\title{
Optimal Network QoS over the Internet of Vehicles for E-Health Applications
}

\author{
Di Lin, ${ }^{1}$ Yuanzhe Yao, ${ }^{1}$ Fabrice Labeau, ${ }^{2}$ Yu Tang, ${ }^{1}$ and Athanasios V. Vasilakos ${ }^{3}$ \\ ${ }^{1}$ School of Information and Software Engineering, University of Electronic Science and Technology of China, Chengdu 610000, China \\ ${ }^{2}$ Electrical and Computer Engineering Department, McGill University, Montreal, QC, Canada H3A 0G4 \\ ${ }^{3}$ Department of Computer Science, Electrical and Space Engineering, Lulea University of Technology, 97187 Lulea, Sweden
}

Correspondence should be addressed to Di Lin; di.lin2@mail.mcgill.ca

Received 11 November 2015; Accepted 26 January 2016

Academic Editor: Lingjie Duan

Copyright (C) 2016 Di Lin et al. This is an open access article distributed under the Creative Commons Attribution License, which permits unrestricted use, distribution, and reproduction in any medium, provided the original work is properly cited.

\begin{abstract}
Wireless technologies are pervasive to support ubiquitous healthcare applications. However, a critical issue of using wireless communications under a healthcare scenario is the electromagnetic interference (EMI) caused by RF transmission, and a high level of EMI may lead to a critical malfunction of medical sensors. In consideration of EMI on medical sensors, we study the optimization of quality of service (QoS) within the whole Internet of vehicles for E-health and propose a novel model to optimize the QoS by allocating the transmit power of each user. Our results show that the optimal power control policy depends on the objective of optimization problems: a greedy policy is optimal to maximize the summation of QoS of each user, whereas a fair policy is optimal to maximize the product of QoS of each user. Algorithms are taken to derive the optimal policies, and numerical results of optimizing QoS are presented for both objectives and QoS constraints.
\end{abstract}

\section{Introduction}

Recent developments in cellular networks have enabled ubiquitous applications of E-health with the aid of medical sensors. However, RF transmission in cellular networks can result in electromagnetic interference (EMI) to medical sensors and a high level of interference can cause malfunction of medical sensors and even injure patients $[1,2]$. Thus, the control of EMI (e.g., through power control) is a critical issue to E-health and should be investigated under the environment of mobile hospital, which is defined as Internet of vehicles for E-health applications throughout this paper. Alternatively, we use the terms of mobile hospital and Internet of vehicles for E-health applications.

1.1. Motivation and Novelty. EMI can cause the malfunction of medical sensors. The network analysis in consideration of EMI control (e.g., through power control) is a critical issue to the E-health networks. However, the algorithms of network analysis in regular wireless networks [3, 4] cannot be employed in E-health networks, since the former does not take into account the impact of EMI on network performance. For example, in E-health network, the wireless users have to reduce their transmit power to control the EMI impact and may not achieve the same level of system capacity estimated by the algorithms used in a regular wireless network.

A large number of works are related to the application of wireless networks in order to support health service $[1,2,5]$. Phunchongharn et al. in $[1,2]$ address the issue of EMI under the scenario of wireless local area networks (WLAN) for E-health applications within a hospital, but the technology of WLAN cannot be applied in our scenario, in which an Internet of vehicles covers a large-scaled area (e.g., a city or a district). Shen et al. in [5] present the possibilities of employing wireless technologies in a medical environment and adjust the level of power and rate with the channel conditions of users. However, the authors do not take the potential EMI impact into account. In such a scenario, a wireless user who is close to a medical sensor could be allowed to transmit data at a high level of power when the user's communication channel is in good condition [6]. However, the RF transmission at 
a high level of power may lead to the malfunction of medical sensors. Such an improper power allocation by the abovementioned algorithms may influence the function of EMIsensitive medical sensors, so these algorithms cannot be employed under the scenario of mobile hospital. Also the above-mentioned algorithms are designed to optimize the individual objective of each wireless user, instead of optimizing a network-level objective (e.g., the quality of network service). The importance of scheduling wireless transmission under a mobile hospital scenario and the lack of efficient algorithms for optimizing network-level objective motivate us to study how wireless users can control their transmit power to achieve certain goals, such as maximizing the quality of network service while ensuring the acceptable level of EMI on medical sensors over Internet of vehicles for E-health applications.

1.2. Main Contributions. In this paper, we present the issue of scheduling wireless transmission under the environment of mobile hospital. The objective of this paper is to optimize certain goals (e.g., the quality of service) at the network level, instead of at the user level. In this paper, we address the problem of optimizing the quality of network service in a mobile hospital environment and propose the algorithm of power control to achieve the optimal network service. To the best of our knowledge, this is the first work which addresses the algorithm of power control to achieve a network-level goal under a wireless network for E-health applications. The primary contributions of this paper are composed of the following issues: (i) establishing a problem of optimizing power control to achieve the globally optimal quality of service at the network level in view of EMI on medical sensors; (ii) analyzing the transmit power and quality of service of each user at the optimal solution to the proposed problem.

\section{Related Work of EMI on Medical Sensors}

In hospital scenarios, the research on EMI begins with the study of immunity of medical equipment to mobile phones. Tan and Hinberg in [7] address that a few pieces of medical equipment are sensitively influenced by the EMI from mobile phones, and the typical pieces of equipment include infusion pumps, ventilators, and ECG monitors. Also, an EMI susceptibility test was conducted by the Medicines and Healthcare Products Regulatory Agency (MHRA) of UK [8]. This test focuses on investigating the EMI of mobile phones within a personal communication network, and its results show that the EMI-sensitive medical equipment include respirators, defibrillators, and external pacemakers. Trigano et al. in [9] and Calcagnini et al. in [10] present how the EMI from GSM mobile phones influences pacemakers and infusion pumps, respectively. The results show that the EMI of mobile phones can lead to the malfunction of both pacemakers and infusion pumps. With the wide use of 3rd-generation (3G) telecommunication systems all over the world, the authors in $[11,12]$ study how EMI impacts medical pieces of equipment within the $3 \mathrm{G}$ bands. Based on the aforementioned research, the International Electrotechnical Committee (IEC), in 2007, publishes the EN60601-1-2 standard, and this standard recommends the level of EMI immunity as $10 \mathrm{~V} / \mathrm{m}$ and $3 \mathrm{~V} / \mathrm{m}$ for non-life-supporting equipment (e.g., defibrillators) and life-supporting equipment (e.g., blood pressure monitors and infusion pumps), respectively. In view of the advances of electromagnetic compatibility (EMC) technologies, Singapore and the UK governments relax the EMI restriction which is recommended by EN60601-1-2 standard, and mobile phones are allowed to be uses in certain areas of hospitals [13]. In consideration of the most advanced EMC of medical equipment, Tang et al. in [14] address an EMI test, which investigates the EMI from GSM900, PCS1800, and 3G mobile communication systems. This test shows that EMI-sensitive equipment includes ECG monitors, audio evoked potential systems, radiographic systems, and ultrasonic fetal heart detectors [14]. From the study results of previous literature, we can reach a conclusion: syringe pumps, ECG monitors, fetal monitors, respirators, anesthesia machines, external pacemakers, infusion pumps, and defibrillators are sensitive to the EMI of mobile phones [15].

The other studies focus on the EMI from devices which have access to a wireless local area network (WLAN), and a typical WLAN usually works around the frequency of $2.4 \mathrm{GHz}$, which is different from the working band of mobile phones. The amount of EMI on medical equipment is partially determined by frequency bands, and thus the research on EMI under the scenario of healthcare monitoring within a WLAN appears. Krishnamoorthy et al. in [16] carry out a measurement of EMI caused by doctor devices on pieces of medical equipment located in three hospitals, and the doctor devices work within a $2.4 \mathrm{GHz}$-band WLAN. The measurements show that the highest level of EMI is $0.552 \mathrm{~V} / \mathrm{m}$, which is in the acceptable EMI range recommended by the EN60601-1-2 standard. However, the test in [16] did not take into account the quality of service (QoS) of data which are transmitted between patient devices and doctor devices. The strategies of restricting the use of mobile phones, such as switching off mobile phones, are not applicable for the scenario of a wireless healthcare monitoring system [17]. In wireless healthcare monitoring systems, doctors and patients need to employ wireless devices to transmit data and establish communication, and the restriction on the level of transmit power may cause a lower level of QoS of data transmission, which would lead to the loss of medical data. Thus, in wireless healthcare monitoring systems, the restriction of transmit power and QoS requirements are usually against each other. Additionally, the simultaneous transmission of data among multiple patient devices and doctor devices would cause a high level of EMI to medical equipment [1]. Phunchongharn et al. in [1] investigate the EMI in hospital environments by considering the QoS of patient devices and doctor devices, and they conclude that the level of EMI on most of the medical equipment is unacceptable when the transmit power of a device is above $10 \mathrm{~mW}$ within a WLAN.

All the above-mentioned research does not consider the vehicular scenarios for healthcare applications, which are interesting to this paper, and thus the medical sensors in the test may not be vehicle-mounted and wearable medical sensors. In Section 3.1, we address a detailed experiment which includes the test of EMI impact on types of vehiclemounted and wearable medical sensors. 


\section{Mobile Hospital Environment}

In a mobile hospital environment, vehicles for E-health applications are mounted with a few medical devices, and these devices can assist doctors in monitoring the patients' condition (to the best of our knowledge, quite a few Internet of medical vehicles examples have already been employed in USA to provide mobile health service. A few real examples of service providers include Mobile Specialty Vehicles and Farber Specialty Vehicles). Under the following scenarios, physicians, nurses, and the patient's relatives may employ mobile phones. (1) Physicians and nurses staff need to keep reporting the patients' conditions over phone to a doctor or healthcare staff in the medical center. Once the patients arrive at the medical center, the staff can arrange the medical actions on this patient. (2) Relatives of patients may need to contact their families or friends by phone about the important information, for example, the variation of clinical situations. However, the nearby medical devices might be impacted by the EMI, which is produced by the use of mobile phones [18]. EMI is defined as the disturbance of electrical circuits caused by electromagnetic radiation from an external source [19], and EMI could lead to loss of data during the transmission.

In the following, we address an experiment of EMI effects on typical medical devices. This experiment is originally proposed in [20], and we present it here for completeness. Then, we establish the model of EMI impact, which is a constraint of our network-capacity optimization problem, detailed in Section 4.

3.1. Experiment of Investigating EMI Impact. In the following experiment, we investigate how the EMI of mobile phones impacts a few typical vehicle-mounted medical devices. For completeness, we choose the mobile phones with quite a few typical technologies, including CDMA2000, TD-LTE, and GSM-900/1800. We carry out this experiment in an anechoic chamber, which can exclude the EMI impact caused by the other RF sources, such as from external communication systems.

The results of experiment show that the EMI from mobile phones could degrade the performance of medical devices when these devices keep a distance of $2 \mathrm{~m}$ from the mobile phones. Typical types of degradation in the experiment include (I) errors occurring in the ultrasound, X-ray, and CT images; (II) artifact in ECG and EEG signals; (III) the malfunction of ventilators, syringe pumps, and infusion pumps; and (IV) irregular operating modes of external pacemakers. The above-mentioned results are in line with the publications of [21-24].

3.2. Model of EMI Impact. Under the scenario of E-health applications, a vehicle is mounted with medical devices, and these devices are either life-support or non-life-support (illustrated in Figure 1). These medical devices first collect medical data and then send these data to physicians or doctors in order to take medical actions on a specific patient once this patient arrives. Also physicians or healthcare staff on the vehicle need to report the latest conditions of a patient to doctors, ensuring that the doctors could acquire the updated

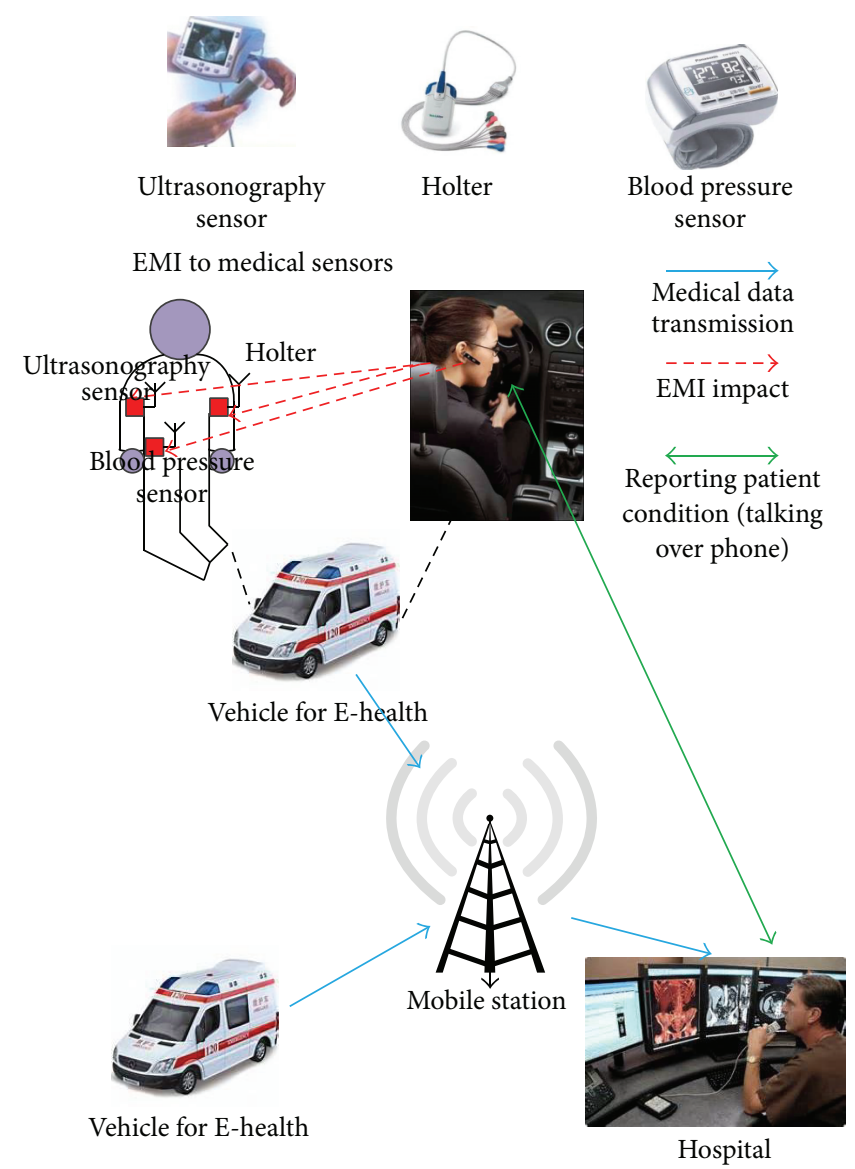

FIGURE 1: The figure illustrates the Internet of vehicles for E-health applications.

patient's conditions. However, nearby medical devices might be impacted by the EMI from the use of mobile phones. Also life-support medical devices are more sensitive to EMI impact than non-life-support devices, since the former contains electronic components, which are EMI-sensitive. Typical lifesupport medical devices are Ultrasonograph devices, and so forth, and typical non-life-support medical devices are blood pressure devices and holters, and so forth.

Different medical devices can allow different levels of transmit power to avoid the malfunction of these devices. To the best of our knowledge, Phunchongharn et al. in [1] firstly present the models of EMI on medical devices and investigate the highest allowable level of transmit power to meet the EMI constraint. The maximal allowable transmit power of a mobile phone needs to satisfy (1) and (2), for non-life-support medical devices and life-support medical devices, respectively [1]:

$$
\begin{aligned}
& \sum_{j \in S} \frac{\theta_{1} \sqrt{P_{j}}}{D_{j}(x)} \leq E_{\mathrm{NL}}(x), \quad \text { for } x \in S_{1}, \\
& \sum_{j \in S} \frac{\theta_{2} \sqrt{P_{j}}}{D_{j}(y)} \leq E_{\mathrm{L}}(y), \quad \text { for } y \in S_{2},
\end{aligned}
$$


where $E_{\mathrm{NL}}(x)$ and $E_{\mathrm{L}}(y)$ are the allowable EMI levels of a nonlife-support device $x$ and a piece of life-support equipment $y$, respectively; $P_{j}$ is the transmit power of a mobile user $j$; $D_{j}(x)$ represents the distance between a non-life-support device $x$ and user $j ; D_{j}(y)$ represents the distance between a life-support device $y$ and user $j ; \theta_{1}$ and $\theta_{2}$ represent two constants, the values of which are recommended by IEC 60601-1-2 as 7 and 23, respectively [1]. $S$ represents the set of mobile users under the scenario of Internet of vehicles. $S_{1}$ is the set of non-life-support devices, while $S_{2}$ is the set of lifesupport devices.

Definition 1. The maximal possible transmit power of user $i$ (i.e., $\bar{P}_{i}$ ) to satisfy the constraint of EMI on medical devices can be computed from (1) and (2). Also we define the minimum of wireless users' allowable transmit power $\bar{P}_{i}$, that is, $P_{\max }=\min _{i} \bar{P}_{i}$, as the maximal effective transmit power (METP) under a mobile hospital scenario (for the detailed process of computing METP refer to [20]).

METP $P_{\max }$ will be employed to establish the optimization problem in Section 4. Each of the mobile users needs to keep his or her transmit power below METP, ensuring an allowable level of EMI under the mobile hospital scenario (in a mobile health scenario, the wireless users may move to any position around a piece of medical equipment, and we assume that each of the users has the same probability to appear at a specific position. So the users close to the same equipment equally share a bounded transmit power. The investigation of differentiated bounds of transmit power for different wireless users (physicians, nurses, patients, and their relatives) would be an interesting topic, but it is out of the scope of this paper).

\section{Optimization of QoS}

In this section, we firstly summarize the mathematical formulation of QoS. Consider an Internet of vehicles with $N$ wireless users. For information user $i$, Signal-to-Noise Ratio (SNR) is defined as

$$
\mathrm{SNR}_{i}=\frac{P_{i} h_{i}}{\sum_{j \neq i} P_{j} h_{j}+I},
$$

where $P_{i}$ denotes the transmit power by user $i ; h_{i}$ denotes the channel condition between user $i$ and base station; $I$ denotes the power of additive white Gaussian noise.

In the following, we will employ SNR as the metric of QoS and discuss how to optimize the QoS within a network of mobile hospital. Given the metric of QoS of each user as (3), we consider the problem of optimizing QoS within a network with two objectives as

$$
\begin{aligned}
& Q_{1}=\sum_{i} \mathrm{SNR}_{i} \\
& \mathrm{Q}_{2}=\prod_{i} \mathrm{SNR}_{i} .
\end{aligned}
$$

The problem objective (5) represents the total throughput maximization with fixed coding. Suppose we have already chosen a specific scheme of coding at the symbol level; that is, we have spreading gain and power at our control. This problem is formulated in [6]. As shown there, the total throughput is always proportional to the level of SINR, where the proportion is determined by the coding scheme. Consequently, optimizing the total throughput equals maximizing the summation of SINR. The problem objective (6) represents the total throughput maximization with flexible coding. Suppose we relaxed the limitation of selecting and fixing a specific scheme of coding at the symbol level. This problem is formulated in [6]. As shown there, the total throughput is always proportional to the level of log function of SINR, where the proportion is determined by the coding scheme. Consequently, optimizing the total throughput equals maximizing the product of SINR. The problem of optimizing total throughput either with fixed coding scheme or with flexible coding scheme is a critical problem in network, and this motivates us to investigate the optimization of system throughput in the scenario of wireless network of E-health, with the design of objectives of (5) and (6).

In the following, we formulate the optimization problems with $Q_{1}$ and $Q_{2}$ as

$$
\begin{array}{ll}
\max _{\mathbf{P}} & \sum_{i} \frac{P_{i} h_{i}}{\sum_{j \neq i} P_{j} h_{j}+I} \\
\text { s.t. } & 0 \leq P_{i} \leq P_{\max } \\
& \mathrm{SNR}_{i}=\frac{P_{i} h_{i}}{\sum_{j \neq i} P_{j} h_{j}+I} \geq \mathrm{SNR}_{\min } \\
& \sum_{i} P_{i} h_{i} \leq P_{S}, \\
\max _{\mathbf{P}} & \prod_{i} \frac{P_{i} h_{i}}{\sum_{j \neq i} P_{j} h_{j}+I} \\
\text { s.t. } & 0 \leq P_{i} \leq P_{\max } \\
& \mathrm{SNR}_{i}=\frac{P_{i} h_{i}}{\sum_{j \neq i} P_{j} h_{j}+I} \geq \mathrm{SNR}_{\min } \\
& \sum_{i} P_{i} h_{i} \leq P_{S},
\end{array}
$$

where $\mathbf{P}$ denotes the optimal transmit power of each user; $P_{\max }$ denotes the maximal possible transmit power among all of the users; $\mathrm{SNR}_{\min }$ denotes the minimal level of SNR which is acceptable to all of the users; $P_{S}$ denotes the total received power constraint. It is due to the fact that the total received power from the data users (for E-health applications) is an interference to other classes of users (not for E-health applications).

With the setting of $x_{i}=P_{i} h_{i}$, we can transform the optimization problems as

$$
\begin{array}{ll}
\max _{\mathbf{P}} & \sum_{i} \frac{x_{i}}{\sum_{j \neq i} x_{j}+I} \\
\text { s.t. } & 0 \leq x_{i} \leq P_{\max } h_{i} \\
& -x_{i}+I \times \mathrm{SNR}_{\min }+\sum_{j \neq i} x_{j} \leq 0 \\
& \sum_{i} x_{i}-P_{S} \leq 0,
\end{array}
$$




$$
\begin{array}{ll}
\max _{\mathbf{P}} & \prod_{i} \frac{x_{i}}{\sum_{j \neq i} x_{j}+I} \\
\text { s.t. } & 0 \leq x_{i} \leq P_{\max } h_{i} \\
& -x_{i}+I \times \mathrm{SNR}_{\min }+\sum_{j \neq i} x_{j} \leq 0 \\
& \sum_{i} x_{i}-P_{S} \leq 0,
\end{array}
$$

where $x_{i}$ denotes the level of power at the receiving end of user $i$.

The Lagrange formulation of optimization problem with objective $Q\left(Q=Q_{1}, Q_{2}\right)$ can be denoted as

$$
\begin{aligned}
L= & Q-\sum_{i} \theta_{i}\left(\frac{x_{i}}{h_{i}}-P_{\max }\right) \\
& -\sum_{i} \lambda_{i}\left(-x_{i}+I \times \mathrm{SNR}_{\min }+\sum_{j \neq i} x_{j}\right) \\
& -\mu\left(\sum_{i} x_{i}-P_{S}\right) \\
= & Q-\sum_{i}\left(\frac{\theta_{i}}{h_{i}}+\mu-\lambda_{i}+\mathrm{SNR}_{\min } \sum_{j \neq i} \lambda_{j}\right) x_{i} \\
& + \text { Const }
\end{aligned}
$$

where $\mu, \lambda_{i}$, and $\theta_{i}$ are the parameters of Lagrange formulation.

Then, the first-order derivative of $L$ is

$$
\begin{aligned}
\frac{\partial L}{\partial x_{i}} & =0 \longrightarrow \\
\frac{\theta_{i}}{h_{i}}+\mu-\lambda_{i}+\mathrm{SNR}_{\min } \sum_{j \neq i} \lambda_{j} & =\frac{\partial Q}{\partial x_{i}} .
\end{aligned}
$$

Note that $P_{S}$ is on all the users, so the value of $\mu$ is common to all the users. We can classify all $N$ users into three disjoint groups according to the binding constraints, that is, depending on the values of $\lambda_{i}$ and/or $\theta_{i}$.

Definition 2. Group 1: $G_{1}=\left\{i \mid \lambda_{i}>0\right\}$, which is equally defined as $G_{1}=\left\{i \mid \mathrm{SNR}_{i}=\mathrm{SNR}_{\min }\right\}$; Group 2: $G_{2}=\{i \mid$ $\left.\lambda_{i}=0, \theta_{i}=0\right\}$, which is equally defined as $G_{2}=\left\{i \mid \mathrm{SNR}_{i}>\right.$ $\left.\mathrm{SNR}_{\min }, P_{i}<P_{\max }\right\}$; Group 3: $G_{3}=\left\{i \mid \lambda_{i}=0, \theta_{i}>0\right\}$, which is equally defined as $G_{3}=\left\{i \mid \mathrm{SNR}_{i}>\mathrm{SNR}_{\min }, P_{i}=P_{\max }\right\}$.

Remark 3. In consideration of EMI and QoS requirements, all of the users cannot violate the constraints of EMI and QoS. Group 1 represents the set of users who are at the verge of violating QoS constraint; Group 3 represents the set of users who are at the verge of violating EMI constraint; Group 2 represents the set of users who are not at the verge of violating any constraint.

\section{Algorithms of Solving Optimization Problems}

In the following, we first derive the general structure of the optimal solution to the problems with objectives $Q_{1}$ and $Q_{2}$, respectively. Then, we address the specific algorithms to solve the optimization problems.

\subsection{Solutions to the Optimal Problem with Objective $Q_{1}$}

Lemma 4. One has $\operatorname{sign}\left(\partial Q_{1} / \partial x_{i}-\partial Q_{1} / \partial x_{j}\right)=\operatorname{sign}\left(x_{i}-x_{j}\right)$, where $\operatorname{sign}(x)$ represents the sign of $x$.

Proof. Given

$$
\frac{\partial Q_{1}}{\partial x_{i}}=\frac{1}{I+\sum_{k=1, k \neq i}^{N} x_{k}}-\sum_{m=1, m \neq k}^{N} \frac{x_{m}}{\left(I+\sum_{k=1, k \neq m}^{N} x_{k}\right)^{2}},
$$

we have

$$
\begin{aligned}
& \frac{\partial Q_{1}}{\partial x_{i}}-\frac{\partial Q_{1}}{\partial x_{j}}=\left(I+\sum_{k=1, k \neq i}^{N} x_{k}\right) \\
& \quad \cdot\left\{\frac{1}{\left(I+\sum_{k=1, k \neq i}^{N} x_{k}\right)^{2}}-\frac{1}{\left(I+\sum_{k=1, k \neq j}^{N} x_{k}\right)^{2}}\right\} .
\end{aligned}
$$

If $x_{i} \leq x_{j}$, we have $\partial Q_{1} / \partial x_{i}-\partial Q_{1} / \partial x_{j} \leq 0$; otherwise, $\partial Q_{1} / \partial x_{i}-\partial Q_{1} / \partial x_{j}<0$. So $\operatorname{sign}\left(\partial Q_{1} / \partial x_{i}-\partial Q_{1} / \partial x_{j}\right)=$ $\operatorname{sign}\left(x_{i}-x_{j}\right)$.

Lemma 5. For an optimum solution, the number of users in group $G_{2}$ is at most one.

Proof. Suppose that we can find two users $i$ and $j$ in group $G_{2}$ which can lead to the optimum $\mathbf{x}^{*}$; then, $\partial Q_{1} / \partial x_{i}^{*}=$ $\partial Q_{1} / \partial x_{j}^{*}=\mu+\sum_{j \notin G_{2}} \lambda_{j}$ given Definition 1 .

Let $\mathbf{x}^{\epsilon}=\left\{x_{1}^{*}, \ldots, x_{i}^{*}+\epsilon, \ldots, x_{j}^{*}-\epsilon, \ldots, x_{N}\right\}$; then, $Q_{1}\left(\mathbf{x}^{\epsilon}\right)-$ $Q_{1}\left(\mathbf{x}^{*}\right)=\int_{0}^{\epsilon}\left(\partial Q_{1} / \partial \epsilon_{0}\right) d \epsilon_{0}>0$, since $\partial Q_{1} / \partial \epsilon_{0}=\left(\partial Q_{1} / \partial x_{i}-\right.$ $\left.\partial Q_{1} / \partial x_{j}\right)\left.\right|_{\mathbf{x}=\mathbf{x}_{0}^{\epsilon}}>0$ for any $0 \leq \epsilon_{0} \leq \epsilon \cdot Q_{1}\left(\mathbf{x}^{\epsilon}\right)>Q_{1}\left(\mathbf{x}^{*}\right)$ is in contradiction with the fact that $\mathbf{x}^{*}$ is the optimum of $Q_{1}$.

Theorem 6. When the number of users in $G_{2}$ is zero, then, we can restrict our attention of an optimal solution to the following cases without losing optimality: any user $j \in G_{3}$ has a better channel condition than any user $i \in G_{1}$ if $P_{i}<P_{\max }$; that is, $h_{i}<h_{j}$ for $j \in G_{3}$ and $i \in G_{1}$ with $P_{i}<P_{\max }$.

Proof. When the number of users in $G_{2}$ is zero, then, any user belongs to $G_{1}$ or $G_{3}$. Suppose user $j \in G_{3}$ and $i \in G_{1}$ with $P_{i}<$ $P_{\text {max }}$, and $\mathbf{x}^{*}=\left\{x_{1}^{*}, \ldots, x_{i}^{*}, \ldots, x_{j}^{*}, \ldots, x_{N}^{*}\right\}$ is the optimum. One has $\partial Q_{1} / \partial x_{i}^{*}-\partial Q_{1} / \partial x_{j}^{*}=-\theta_{j} / h_{j}-\left(1+\mathrm{SNR}_{\min }\right) \lambda_{i}<0$. By Lemma 4 , we have $x_{i}^{*}<x_{j}^{*}$.

Let $\mathbf{x}^{* *}=\left\{x_{1}^{*}, \ldots, x_{j}^{*}, \ldots, x_{i}^{*}, \ldots, x_{N}^{*}\right\}$, that is; we exchange the position of $i$ th and $j$ th items of $\mathbf{x}^{*}$. Assume $h_{i} \geq h_{j}$. Since $x_{i}^{*}<x_{j}^{*}$ and $Q_{1}\left(\mathbf{x}^{* *}\right)=Q_{1}\left(\mathbf{x}^{*}\right)$, we know that $\mathbf{x}^{* *}$ is also an optimal solution to problem (6). However, $\mathbf{x}^{* *}$ has a user $j$ who belongs to $G_{2}$. Thus, the assumption of $h_{i} \geq h_{j}$ leads to a contradiction. 
Theorem 7. When the number of users in $G_{2}$ is 1 , the users with worse channel condition than the users in $G_{2}$ must belong to $G_{1}$; that is, any user $i$ with $h_{i}<h_{j}$ given $j \in G_{2}$ satisfies $i \in G_{1}$.

Proof. Suppose that $\mathbf{x}^{*}=\left\{x_{1}^{*}, \ldots, x_{i}^{*}, \ldots, x_{j}^{*}, \ldots, x_{N}^{*}\right\}$ is the optimum. Consider users $j \in G_{3}$ and $i \in G_{2}$. One has $\partial Q_{1} / \partial x_{i}^{*}-\partial Q_{1} / \partial x_{j}^{*}=-\theta_{j} / h_{j}<0$. By Lemma 4, we have $x_{i}^{*}<x_{j}^{*}$.

Assume $h_{i} \geq h_{j}$. Following the same way as that used in the proof of Theorem 6 , by exchanging the position of $i$ th and $j$ th items of $\mathbf{x}^{*}$, we can find an optimum $\mathbf{x}^{* *}$ with two users who belong to $G_{2}$. Thus, the assumption of $h_{i} \geq h_{j}$ leads to a contradiction.

All of users in $G_{3}$ have a better channel condition than the user in $G_{2}$. Thus, if a user $k$ with $h_{k}<h_{i}$, given $i \in G_{2}$, then, user $k$ belongs to $G_{1}$.

Theorem 8. When the number of users in $G_{2}$ is 1 , we can restrict our attention of an optimal solution to the following cases without losing optimality: all the users with better channel condition than the user in $G_{2}$ belong to $G_{3}$; that is, any user $i$ with $h_{i}>h_{j}$ given $j \in G_{2}$ satisfies $i \in G_{3}$.

Proof. Refer to Theorem 7.

Remark 9. The optimal solution to the problem with $Q_{1}$ implies a greedy policy: the user $i$ with better channel conditions (a higher $h_{i}$ ) is allocated to $P_{\max }$ (in group $G_{3}$ ), the user $i$ with worse channel conditions (a lower $h_{i}$ ) is allocated to a level of power which can only meet the minimal QoS (in group $G_{1}$ ), and the number of users in $G_{2}$ is at most one.

\subsection{Solutions to the Optimal Problem with Objective $Q_{2}$}

Lemma 10. One has $\operatorname{sign}\left(\partial Q_{2} / \partial x_{i}-\partial Q_{2} / \partial x_{j}\right)=-\operatorname{sign}\left(x_{i}-\right.$ $\left.x_{j}\right)$, where $\operatorname{sign}(x)$ represents the sign of $x$.

Proof. Given

$$
\frac{\partial Q_{2}}{\partial x_{i}}=Q_{2}\left\{\frac{1}{x_{i}}-\sum_{m=1, m \neq i}^{N} \frac{1}{\left(I+\sum_{k=1, k \neq m}^{N} x_{k}\right)}\right\},
$$

we have

$$
\frac{\partial Q_{2}}{\partial x_{i}}-\frac{\partial Q_{2}}{\partial x_{j}}=\frac{1}{x_{i}\left(I+x_{i}\right)}-\frac{1}{x_{j}\left(I+x_{j}\right)}
$$

If $x_{i} \leq x_{j}$, we have $\partial Q_{2} / \partial x_{i}-\partial Q_{2} / \partial x_{j} \geq 0$; otherwise, $\partial Q_{2} / \partial x_{i}-\partial Q_{2} / \partial x_{j}>0$. So $\operatorname{sign}\left(\partial Q_{2} / \partial x_{i}-\partial Q_{2} / \partial x_{j}\right)=$ $-\operatorname{sign}\left(x_{i}-x_{j}\right)$.

Theorem 11. For an optimum solution, if there are users in $G_{2}$, their level of power at the receiving end should be the same. In other words, if there are users $i, j \in G_{2}$, then $x_{i}=x_{j}$.

Proof. Consider users $i, j \in G_{2}$. We have $\partial Q_{2} / \partial x_{i}=$ $\partial Q_{2} / \partial x_{j}=\mu+\mathrm{SNR}_{\min } \sum_{k \in A_{1}} \lambda_{k}$. So $x_{i}=x_{j}$ by Lemma 10.
Theorem 12. Except for the case when all the users' powers at the receiving end $x_{i}(i=1,2, \ldots, N)$ are the same, if there are users in $G_{1}$, then these users should be allocated the maximal transmit power, that is, $P_{\max }$.

Proof. Suppose that $\mathbf{x}^{*}=\left\{x_{1}^{*}, \ldots, x_{i}^{*}, \ldots, x_{j}^{*}, \ldots, x_{N}^{*}\right\}$ is the optimum. Consider users $i \in G_{1}$ and $j \notin G_{1}$. Assume $P_{i}<P_{\max }$. Then, we have $\partial Q_{2} / \partial x_{i}-\partial Q_{2} / \partial x_{j}<0$, and thus $x_{i}>x_{j}$ by Lemma 10. However, from the monotonicity of $\mathrm{SNR}$ equation shown in (3), we have $\mathrm{SNR}_{i}=\mathrm{SNR}_{\text {min }}>\mathrm{SNR}_{j}$ which is a contradiction. Thus, we have $P_{i}=P_{\max }$.

Theorem 13. Except for the case when all the users' powers at the receiving end $x_{i}(i=1,2, \ldots, N)$ are the same, there can be at most one user in $G_{1}$ and that user is with the worst channel condition $h_{i}$. In other words, the number of users in $G_{1}$ is at most 1 , and $h_{i}$ is lowest if $i \in G_{1}$.

Proof. Suppose that $\mathbf{x}^{*}=\left\{x_{1}^{*}, \ldots, x_{i}^{*}, \ldots, x_{j}^{*}, \ldots, x_{N}^{*}\right\}$ is the optimum. Assume that there are two users $i, j \in G_{1}$ with $h_{i}>$ $h_{j}$. By Theorem 12, $P_{i}=P_{j}=P_{\max }$. So we have $x_{i}>x_{j}$, and $\mathrm{SNR}_{i}>\mathrm{SNR}_{j}$ from the monotonicity of SNR equation, which is a contradiction with $\mathrm{SNR}_{i}=\mathrm{SNR}_{j}=\mathrm{SNR}_{\min }$. So there is at most one user in $G_{1}$.

Theorem 14. For an optimum solution, if there exists user $i$ in $G_{3}$, then $x_{i}$ should be less than $x_{j}$ for any user $j \in G_{2}$.

Proof. Suppose that $\mathbf{x}^{*}=\left\{x_{1}^{*}, \ldots, x_{i}^{*}, \ldots, x_{j}^{*}, \ldots, x_{N}^{*}\right\}$ is the optimum. Consider users $i \in G_{3}$ and $j \in G_{2}$. One has $\partial Q_{2} / \partial x_{i}^{*}-\partial Q_{2} / \partial x_{j}^{*}=\theta_{j} / h_{j}>0$. By Lemma 10, we have $x_{i}^{*}<x_{j}^{*}$.

Remark 15. The optimal solution to the problem with $Q_{2}$ implies a fair policy: the user $i$ with worse channel conditions (a lower $h_{i}$ ) is allocated to $P_{\max }$ (in group $G_{1}$ or $G_{3}$ ); the user $i$ with better channel conditions (a higher $h_{i}$ ) is allocated to the same level of power at the receiving end $x_{i}$, which is higher than $x_{j}$ of user $j$, who is with lower priority (a lower $h_{j}$ ).

\subsection{Algorithms of Solving the Optimization Problem}

Theorem 16. For problem of (6), without losing optimality, we can focus our attention on an optimal power allocation vector to the following form: $\mathbf{P}^{*}=\left\{P_{\max }, \ldots, P_{\max }, P_{T}, P_{T+1}, \ldots, P_{N}\right\}$, where $T$ is an integer and $1 \leq T \leq N ; 0 \leq P_{T} \leq P_{\max }$; $\mathrm{SNR}_{i}=$ $\mathrm{SNR}_{\min }$ for any $T<i \leq N$.

Proof. Refer to Remark 3 and Theorems 6 to 8 .

From Theorem 16, an optimal solution to problem (6) can be found as follows. Partition the users into two classes: $T$ low-priority users and $(N-T)$ high-priority users, based on their channel conditions $h_{i}(i=1,2, \ldots, N$ ) (a user in a better channel condition, i.e., a larger value of $h_{i}$, is allocated a higher priority). For each of the $N+1$ possible ways of partitioning users, allocate the power in the following way. Allocate $P_{\max }$ to all high-priority users while the power is allocated to all low-priority users just to meet the QoS 


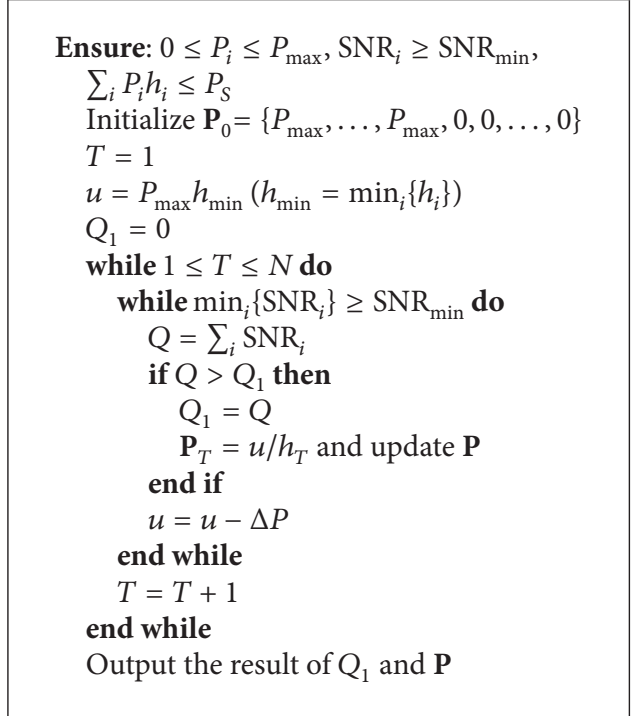

Algorithm 1: Algorithm of solving optimization problem (6).

requirement, that is, $\mathrm{SNR}_{\min }$. Optimize the objective with respect to the variable $T$, subject to the constraints of (6). Evaluate the objective and compare the values of objective for each possible partitioning rule. Pick $T$ that yield the optimum by increasing $u$ at a step of $\Delta P$. The specific algorithm is shown in Algorithm 1.

Remark 17. Algorithm 1 solves the problem of (6) within the running time of $O(N K)$ where $K=\left\lceil P_{\max } h_{\min } / \Delta P\right\rceil$ and $h_{\min }=\min _{i}\left\{h_{i}\right\}$.

Remark 18. Exploratory search algorithms solve the problem of (6) within the running time of $O\left(K^{N}\right)$ where $K=$ $\left\lceil P_{\max } h_{\min } / \Delta P\right\rceil$.

Theorem 19. For problem of (7), without losing optimality, we can focus our attention on an optimal power allocation vector to the following form: $\mathbf{P}^{*}=\left\{P_{1}, \ldots, P_{T}, P_{\max }, \ldots, P_{\max }\right\}$, where $T$ is an integer and $1 \leq T \leq N ; 0 \leq P_{T} \leq P_{\max } ; x_{1}=x_{2}=\cdots=$ $x_{T} \geq x_{T+1}=P_{\max } h_{T+1}$.

Proof. Refer to Remark 3 and Theorems 11 to 14.

From Theorem 19, an optimal solution to problem (7) can be found as follows. Partition the users into two classes: $T$ low-priority users and $(N-T)$ high-priority users, based on their channel conditions $h_{i} i=1,2, \ldots, N$. For each of the $N+1$ possible ways of partitioning users, allocate the power in the following way. Allocate $P_{\max }$ to all low-priority users while the power is allocated among high-priority users to ensure that their $x_{i}(i=T+1, \ldots, N)$ is the same $\left(x_{i}=\right.$ $u(i=T+1, \ldots, N))$ and higher than $P_{\max } h_{j}(j=1, \ldots, T)$. Optimize the objective with respect to two variables $T$ and $u$, subject to all the constraints. Evaluate the objective and compare the values of objective for each possible partitioning rule. Pick $T$ and $u$ that yield the optimum by increasing $u$ at a step of $\Delta P$. The specific algorithm is shown in Algorithm 2.

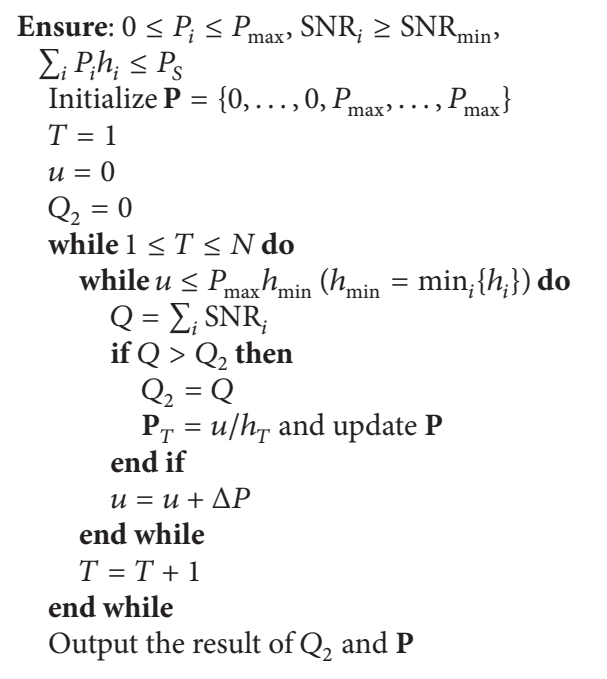

Algorithm 2: Algorithm of solving optimization problem (7).

Remark 20. Algorithm 2 solves the problem of (7) within the running time of $O(N K)$ where $K=\left\lceil P_{\max } h_{\min } / \Delta P\right\rceil$.

Remark 21. Exploratory search algorithms solve the problem of (7) within the running time of $O\left(K^{N}\right)$ where $K=$ $\left\lceil P_{\max } h_{\min } / \Delta P\right\rceil$.

\section{Simulation Results}

We collect the Internet-of-vehicles data from [26], in which a transmit-receive pair of mobile users is represented by a connection of network. In the following simulation, we set 50 nodes in the vehicle network, each node using a mobile phone with a probability of 0.1 . Please note that 50 mobile terminals can produce EMI impact on medical devices at the same time in a densely populated city. Also we set the average distance between mobile users as 8 meters. Each of the users can move at a speed of $10 \mathrm{~m} / \mathrm{s}(36 \mathrm{~km} / \mathrm{h})$ in an arbitrary direction. We clarify the model of channel characteristics in Section 5.1 and perform 100000 Matlab-based runs in the experiment to address the results. The levels of EMI $E_{\mathrm{LS}}$ or $E_{\mathrm{NLS}}$ (see (1) and (2)) are normalized to unity in the simulation.

6.1. Characteristics of Channel Models. We firstly select a few typical empirical channel models for simulation, and these models are presented in ITU-R recommendation M.1225 [25]. The ITU-R M.1225 model can be applied under the scenarios of mobile hospitals outside the high-rise core of urban areas, in which the height of buildings is almost uniform [25]. Mathematically, the channel characteristics $L$ can be addressed as

$$
\begin{aligned}
L= & 40\left(1-4 \times 10^{-3} \Delta h\right) \log D-18 \log \Delta h+21 \log c \\
& +80,
\end{aligned}
$$

where $D[\mathrm{~km}]$ refers to the distance between base station and mobile terminals; $c[\mathrm{MHz}]$ is the frequency of carriers; 


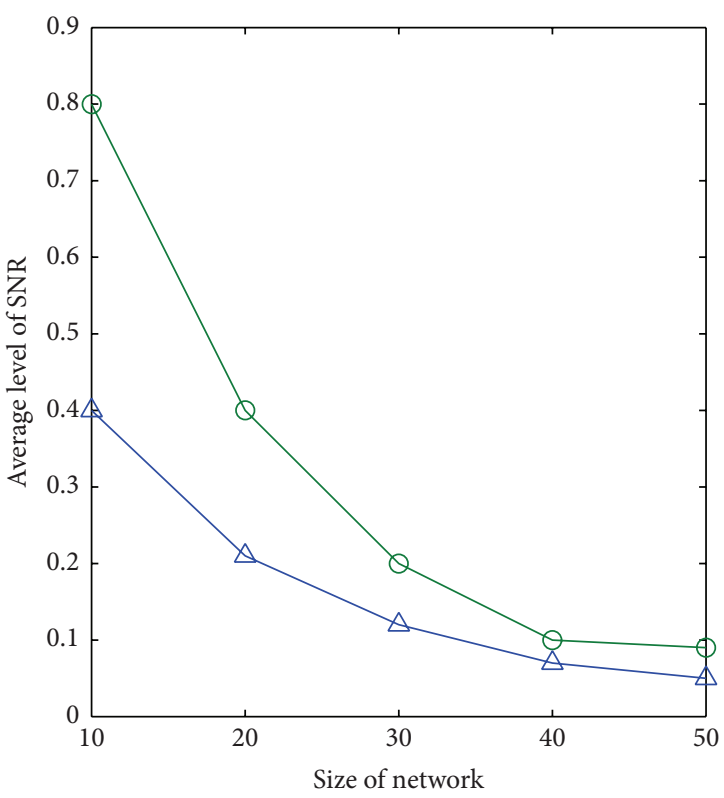

(a)

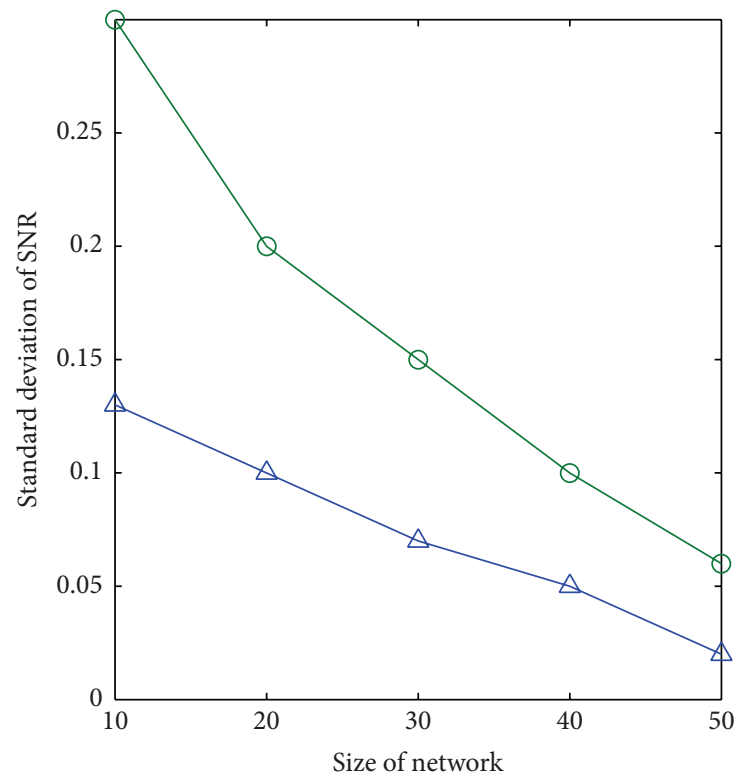

(b)

Figure 2: The figure illustrates the change of SNR with various sizes of networks $N$ (objective of $Q_{1}$ versus objective of $Q_{2}$ ). (a) Average level of SNR; (b) standard deviation of SNR. Green line with "O" represents the problem with objective $Q_{1}$; blue line with " $\triangle$ " represents the problem with objective $Q_{2}$.

$h[\mathrm{~m}]$ refers to the height of antenna at the base station; and the height is measured from an average roof-top level.

The authors in [25] characterize each terrestrial environment as a channel impulse response by using tapped-delay lines. Specifically, the model is composed of quite a few taps: the time delay is determined by the first tap; the average level of power is determined by the strongest tap. Table 1 addresses the propagation model for all of vehicular experiment cases. In each of these cases, Table 1 lists the strength of signals, the relative time delay, and Doppler shift. Specifically, the primary parameters of characterizing propagation models are

(i) time delay: the structure of spread, and its probability distribution;

(ii) multipath fading characteristics: Doppler spectrum in Rician channels versus in Rayleigh channels.

6.2. QoS in the Network of Mobile Hospital. In this section, we address the average level of SNR in a network of mobile hospital as well as the difference among individual SNR levels with different objectives of optimizing QoS. Also we investigate how the parameters of network size $N$, the maximal possible transmit power $P_{\max }$, and the power of noise $I$ can impact the SNR level.

We set $P_{\max }=1$ and $I=10$. It is observed from Figure 2 that the optimization problem with objective $Q_{1}$ can achieve a higher level of average SNR than that with objective $Q_{2}$, while the latter can achieve a much lower level of SNR difference among users. This is because the optimal solution to the problem with $Q_{1}$ implies a greedy policy: the users in better channel conditions (i.e., the users with a higher $h_{i}$ ) are allocated to $P_{\max }$ while the users in worse channel
TABLE 1: Parameters of propagation models in ITU-R recommendation M.1225 [25].

\begin{tabular}{cccc}
\hline Tap & $\begin{array}{c}\text { Relative } \\
\text { delay }(\mathrm{ns})\end{array}$ & $\begin{array}{c}\text { Average } \\
\text { power }(\mathrm{dB})\end{array}$ & $\begin{array}{c}\text { Doppler } \\
\text { spectrum }\end{array}$ \\
\hline 1 & 0 & 0.0 & Rayleigh \\
2 & 310 & -1.0 & Rayleigh \\
3 & 710 & -9.0 & Rayleigh \\
4 & 1090 & -10.0 & Rayleigh \\
5 & 1730 & -15.0 & Rayleigh \\
6 & 2510 & -20.0 & Rayleigh \\
\hline
\end{tabular}

conditions are allocated to a low level of power which can only meet the minimal level of QoS. However, the optimal solution to the problem with $Q_{2}$ implies a fair policy: the users in worse channel conditions (i.e., the users with a lower $h_{i}$ ) are allocated to $P_{\max }$. Thus, the difference of SNR among users with objective $Q_{2}$ is much lower than that with objective $Q_{1}$.

We set $N=50$. It is observed from Figure 3 that the optimization solution is influenced by both the parameters of $P_{\max }$ and $I$. With the increase of $P_{\max }$, the average level of SNR increases first and then decreases, suggesting that there is a threshold of $P_{\max }$ beyond which noise has a more significant impact on SNR than signal does. Unsurprisingly, with the increase of $I$ (i.e., the decrease of $1 / I$ ), the value of SNR also decreases. However, the impact of high values of $I$ (low values of $1 / I$ ) on the SNR decreases significantly as the $I$ increases suggesting that there is a threshold of noise power $I$ below which additional increase of average level of SNR ceases to be advisable. 


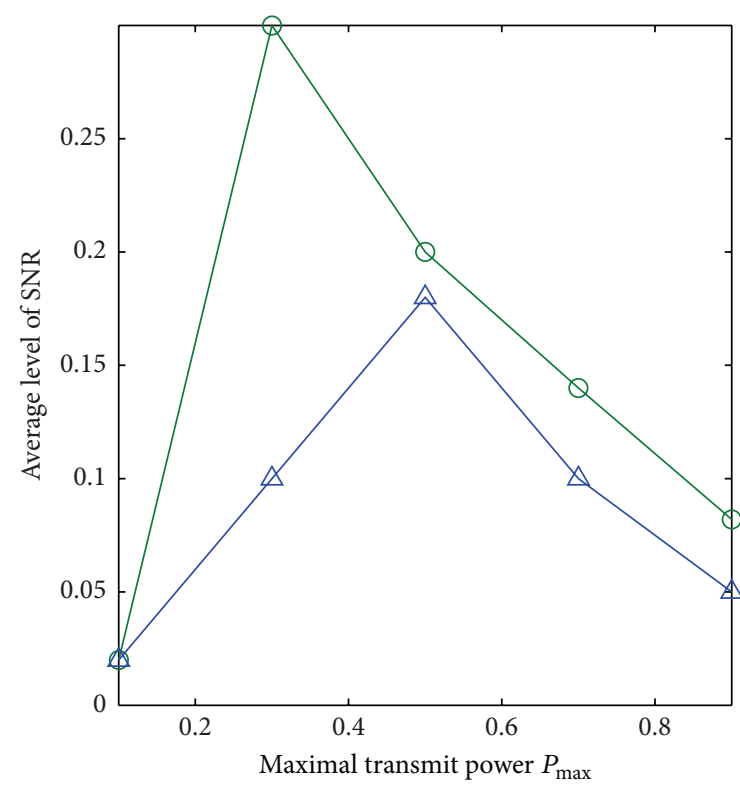

(a)

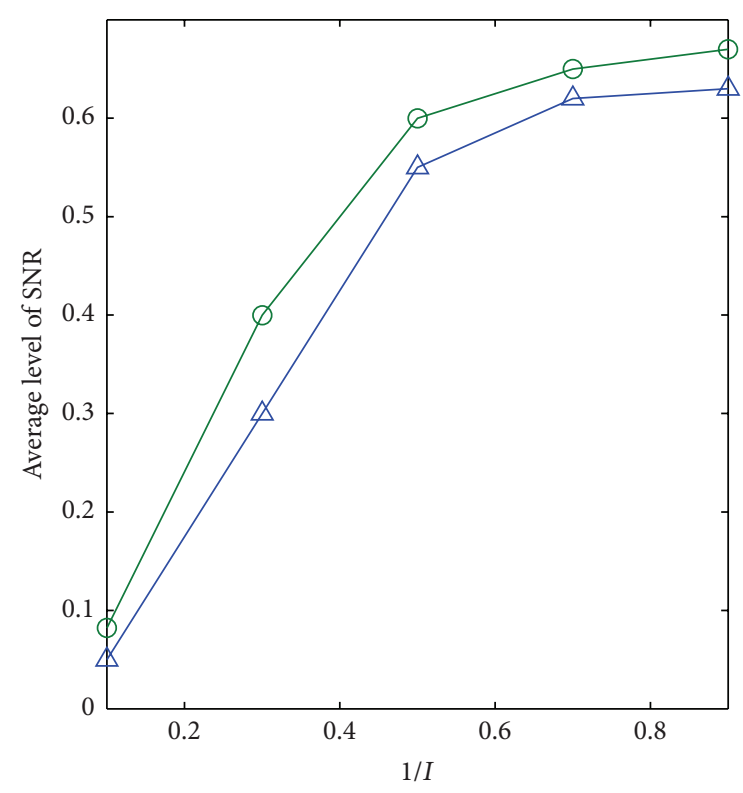

(b)

FIGURE 3: The figure illustrates the change of SNR with parameters of $P_{\max }$ and $I$ (objective of $Q_{1}$ versus objective of $Q_{2}$ ). (a) Average level of SNR versus $P_{\max }$; (b) average level of SNR versus $I$. Green line with "O" represents for the problem with objective $Q_{1}$; blue line with " $\triangle$ " represents for the problem with objective $Q_{2}$.

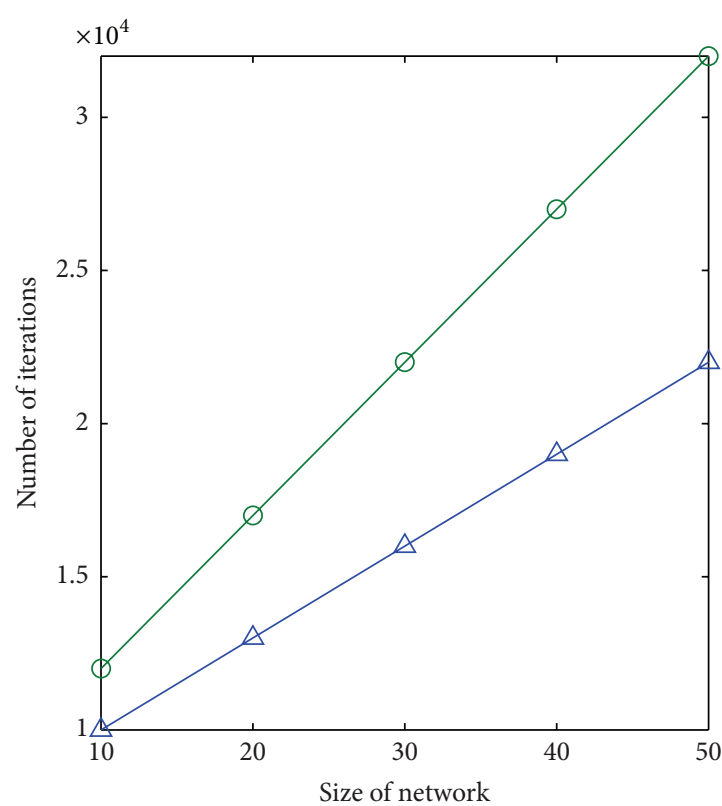

(a)

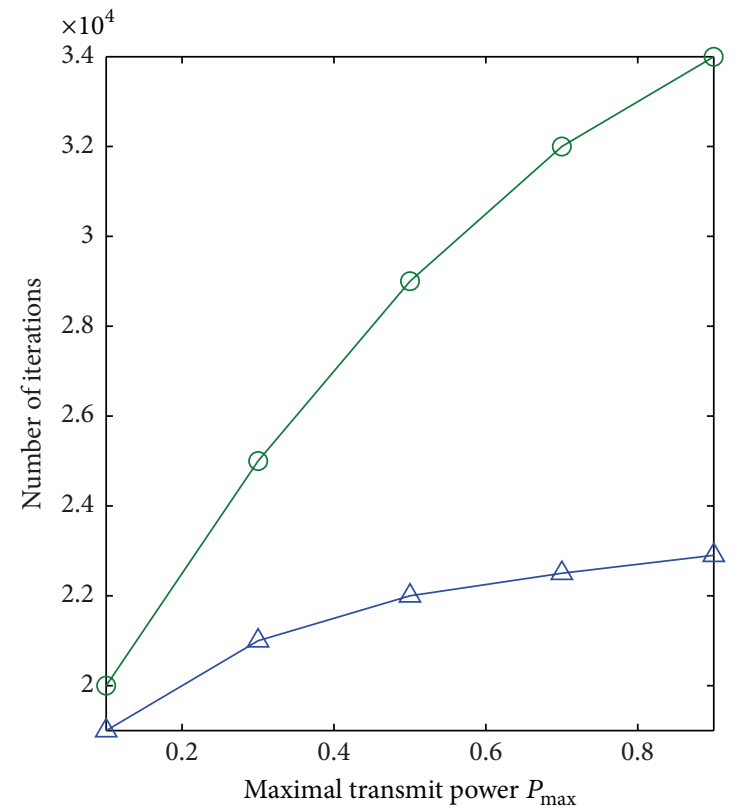

(b)

FIGURE 4: The figure illustrates the convergence rate with parameters of network size $N$ and $P_{\max }$ (objective of $Q_{1}$ versus objective of $Q_{2}$ ). (a) Convergence rate versus $N$; (b) convergence rate versus $P_{\max }$. Green line with "O" represents for the problem with objective $Q_{1}$; blue line with " $\triangle$ " represents for the problem with objective $Q_{2}$.

6.3. Convergence of Optimization Algorithms. In this section, we address the convergence rate of our optimization algorithms by investigating how the parameters of network size $N$ and the maximal possible transmit power $P_{\max }$ can impact the convergence rate.
It is observed from Figure 4 that the algorithm for the problem with objective $Q_{2}$ quickly converges to the optimal solution, while the algorithm for the problem with objective $Q_{1}$ converges to the optimal solution at a low rate. Indeed, the former can reach the optimum after $3.4 \times 10^{4}$ iterations, while 


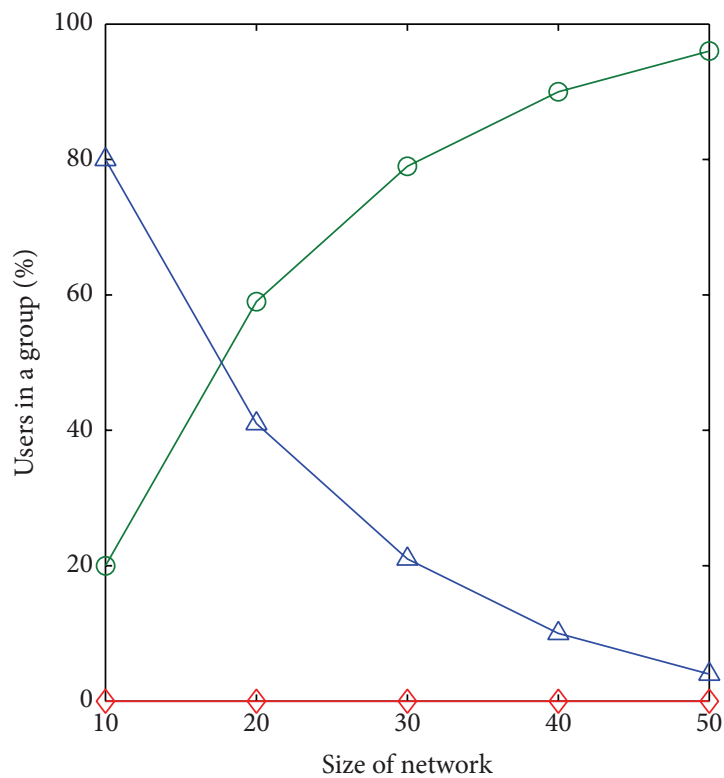

(a)

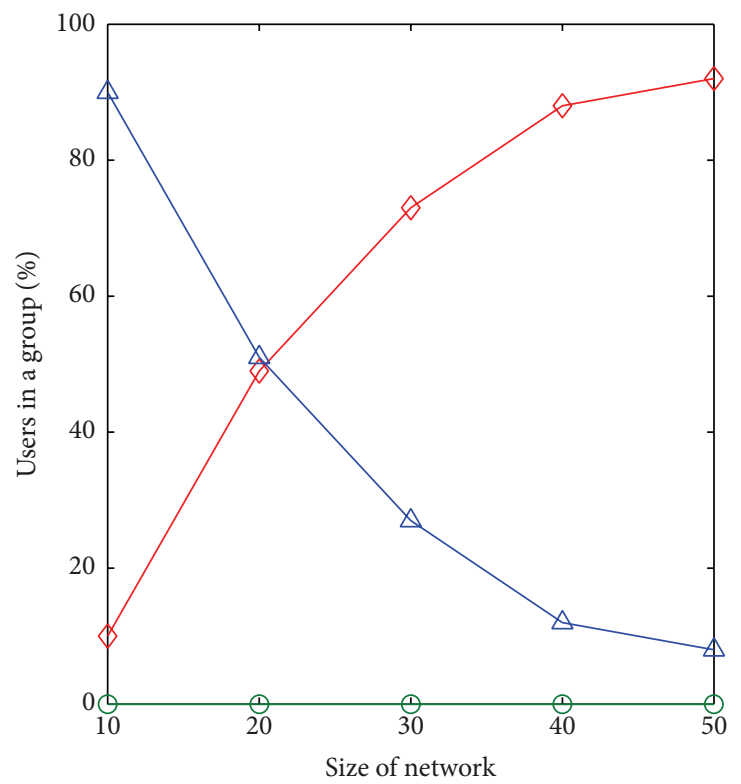

(b)

Figure 5: The figure illustrates the distribution of users in each group with network size $N$. (a) Distribution of users with objective $Q_{1}$; (b) distribution of users with objective $Q_{2}$. Green line with "O" represents the percentage of users in $G_{1}$; red line with " $\diamond$ " represents the percentage of users in $G_{2}$; blue line with " $\triangle$ " represents the percentage of users in $G_{3}$.

the latter convergence appears after $2.3 \times 10^{4}$ iterations under a network of mobile hospital at a scale of 50 .

6.4. Distribution of Users among Three Groups. In this section, we present the distribution of users among three groups at the optimum and investigate the impact of optimization policies (the objectives of optimization problems) on the distribution.

It is observed from Figure 5 that the distribution of users in three groups is quite different for the objective of $Q_{1}$ and $Q_{2}$. In the optimal solution to the problem with objective $Q_{1}$, the percentage of users in $G_{1}$ increases dramatically while the percentage of users in $G_{3}$ decreases dramatically with the rise of $N$. The percentage of users in $G_{2}$ is close to 0 . In the optimal solution to the problem with objective $Q_{2}$, the percentage of users in $G_{2}$ increases dramatically while the percentage of users in $G_{3}$ decreases dramatically with the rise of $N$. The percentage of users in $G_{1}$ is close to 0 .

In the results for both objectives $Q_{1}$ and $Q_{2}$, the percentage of users in $G_{3}$ decreases with the rise of $N$. In other words, the base station starts to reduce the transmit power of most users. This is because a large number of users can lead to a great amount of interference to any user and thus the base station must decrease the transmit power to reduce the interference within the whole network.

\section{Conclusion}

We consider the setting of optimizing QoS in a network of mobile hospital, in which a user receives both signal from base station and the noise from the other users. With the QoS metric (i.e., SNR), we study the optimization of QoS within the whole network and propose a novel algorithm to allocate the transmit power for the maximum of SNR. Some of the key inferences drawn are

(i) the problems with two proposed objectives can yield to two policies of optimizing QoS: a greedy policy is optimal to maximize the sum of SNR of each user, whereas a fair policy is optimal to maximize the product of SNR of each user;

(ii) proposed SNR optimization algorithm can achieve the optimum within a running time which linearly increases with the size of network $N$;

(iii) the analysis on SNR optimization shows the partition of users into three groups with certain features and how the parameters of network impact the distribution of users among these groups.

We would also like to extend our results to a setting which contains multiple priorities of users, and, in such a setting, a few users in the higher priority may have more rigorous QoS requirements than low-priority users. Also we would like to investigate how to design QoS-optimization policies under a setting when users are noncooperative and each user may reject the allocation of transmit power with a certain probability.

\section{Conflict of Interests}

The authors declare that there is no conflict of interests regarding the publication of this paper. 


\section{Acknowledgments}

This work was partially supported by the National Natural Science Foundation of China (no. 61370202), partially supported by a grant from the National High Technology Research and Development Program of China (863 Program, no. 2012AA02A614), and partially supported by the Fundamental Research Funds for the Central Universities (no. ZYGX2015J071).

\section{References}

[1] P. Phunchongharn, D. Niyato, E. Hossain, and S. Camorlinga, "An EMI-aware prioritized wireless access scheme for e-Health applications in hospital environments," IEEE Transactions on Information Technology in Biomedicine, vol. 14, no. 5, pp. 12471258, 2010.

[2] P. Phunchongharn, E. Hossain, and S. Camorlinga, "Electromagnetic interference-aware transmission scheduling and power control for dynamic wireless access in hospital environments," IEEE Transactions on Information Technology in Biomedicine, vol. 15, no. 6, pp. 890-899, 2011.

[3] Y. L. Che, R. Zhang, Y. Gong, and L. Duan, "On spatial capacity of wireless ad hoc networks with threshold based scheduling," IEEE Transactions on Wireless Communications, vol. 13, no. 12, pp. 6915-6927, 2014.

[4] Y. L. Che, R. Zhang, Y. Gong, and L. Duan, "Robust optimization of cognitive radio networks powered by energy harvesting," in Proceedings of the 34th IEEE International Conference on Computer Communications (INFOCOM '15), Hong Kong, April-May 2015.

[5] Q. Shen, X. Liang, X. Shen, X. Lin, and H. Y. Luo, "Exploiting geo-distributed clouds for a E-health monitoring system with minimum service delay and privacy preservation," IEEE Journal of Biomedical and Health Informatics, vol. 18, no. 2, pp. 430-439, 2014.

[6] M. R. Javan and A. R. Sharafat, "Efficient and distributed SINRBased joint resource allocation and base station assignment in wireless CDMA networks," IEEE Transactions on Communications, vol. 59, no. 12, pp. 3388-3399, 2011.

[7] K.-S. Tan and I. Hinberg, "Radiofrequency susceptibility tests on medical equipment," in Proceedings of the 16th Annual International Conference of the IEEE Engineering in Medicine and Biology Society, Engineering Advances: New Opportunities for Biomedical Engineers, vol. 2, pp. 998-999, Baltimore, Md, USA, November 1994.

[8] "Electromagnetic compatibility of medical devices with mobile communications," in Medical Devices Bulletin DB9702, Medical Devices Agency, London, UK, 1997.

[9] A. J. Trigano, A. Azoulay, M. Rochdi, and A. Campillo, "Electromagnetic interference of external pacemakers by walkie-talkies and digital cellular phones: experimental study," Pacing and Clinical Electrophysiology, vol. 22, no. 4, pp. 588-593, 1999.

[10] G. Calcagnini, P. Bartolini, M. Floris et al., "Electromagnetic interference to infusion pumps from GSM mobile phones," in Proceedings of the 26th Annual International Conference of the IEEE Engineering in Medicine and Biology Society (IEMBS '04), vol. 2, pp. 3515-3518, IEEE, San Francisco, Calif, USA, September 2004.

[11] Y. Chu and A. Ganz, "A mobile teletrauma system using 3G networks," IEEE Transactions on Information Technology in Biomedicine, vol. 8, no. 4, pp. 456-462, 2004.
[12] E. A. V. Navarro, J. R. Mas, J. F. Navajas, and C. P. Alcega, "Performance of a 3G-based mobile telemedicine system," in Proceedings of the 3rd IEEE Consumer Communications and Networking Conference (CCNC '06), vol. 2, pp. 1023-1027, IEEE, Las Vegas, Nev, USA, January 2006.

[13] J. Shepherd, 2009, http://www.theguardian.com/society/2009/ jan/06/mobile-phones-hospitals.

[14] C.-K. Tang, K.-H. Chan, L.-C. Fung, and S.-W. Leung, "Electromagnetic interference immunity testing of medical equipment to second- and third-generation mobile phones," IEEE Transactions on Electromagnetic Compatibility, vol. 51, no. 3, pp. 659664, 2009.

[15] M. Ardavan, K. Schmitt, and C. W. Trueman, "A preliminary assessment of EMI control policies in hospitals," in Proceedings of the 14th International Symposium on Antenna Technology and Applied Electromagnetics and the American Electromagnetics Conference (ANTEM/AMEREM '10), pp. 1-6, Ottawa, Canada, July 2010.

[16] S. Krishnamoorthy, J. H. Reed, C. R. Anderson, P. N. Robert, and S. Srikanteswara, "Characterization of the $2.4 \mathrm{GHz}$ ISM band electromagnetic interference in a hospital environment," in Proceedings of the 25th Annual International Conference of the IEEE Engineering in Medicine and Biology Society (EMBC '03), vol. 4, pp. 3245-3248, IEEE, Buenos Aires, Argentina, September 2003.

[17] D. Witters and S. Seidman, "EMC and wireless healthcare," in Proceedings of the Asia-Pacific Symposium on Electromagnetic Compatibility, Beijing, China, April 2010.

[18] S. G. Myerson, "Mobile phones in hospitals are not as hazardous as believed and should be allowed at least in non-clinical areas," British Medical Journal, vol. 326, no. 7387, pp. 460-461, 2003.

[19] F. Fiori, "Integrated circuit susceptibility to conducted RF Interference," Compliance Engineering, vol. 17, pp. 40-49, 2014.

[20] D. Lin, X. Wu, F. Labeau, and A. Vasilakos, "Internet of vehicles for E-health applications in view of EMI on medical sensors," Journal of Sensors, vol. 2015, Article ID 315948, 10 pages, 2015.

[21] W. D. Kimmel and D. D. Gerke, Ten Common EMI Problems in Medical Electronics, 2005, http://www.medicalelectronicsdesign .com/article/ten-common-emi-problems-medical-electronics.

[22] G. Acampora, D. J. Cook, P. Rashidi, and A. V. Vasilakos, "A survey on ambient intelligence in healthcare," Proceedings of the IEEE, vol. 101, no. 12, pp. 2470-2494, 2013.

[23] D. He, C. Chen, S. Chan, J. Bu, and A. V. Vasilakos, "ReTrust: attack-resistant and lightweight trust management for medical sensor networks," IEEE Transactions on Information Technology in Biomedicine, vol. 16, no. 4, pp. 623-632, 2012.

[24] N. Xiong, A. V. Vasilakos, L. T. Yang et al., "Comparative analysis of quality of service and memory usage for adaptive failure detectors in healthcare systems," IEEE Journal on Selected Areas in Communications, vol. 27, no. 4, pp. 495-509, 2009.

[25] ITU-R Recommendation M.1225, “Guidelines for evaluation of radio transmission technologies for IMT-2000," Question ITUR 39/8, 1997.

[26] J. Leskovec, K. J. Lang, A. Dasgupta, and M. W. Mahoney, "Community structure in large networks: natural cluster sizes and the absence of large well-defined clusters," Internet Mathematics, vol. 6, no. 1, pp. 29-123, 2009. 

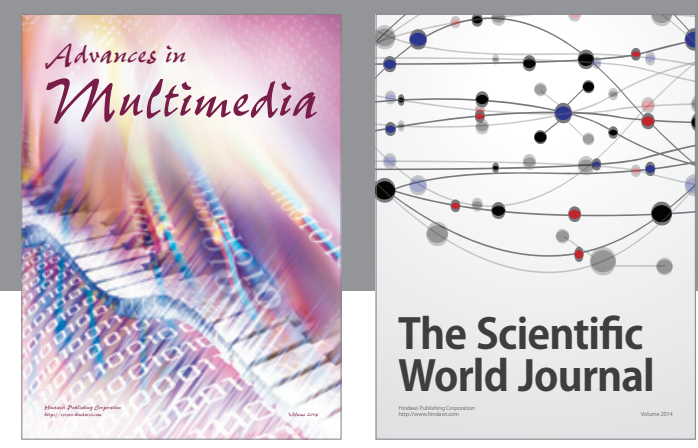

The Scientific World Journal
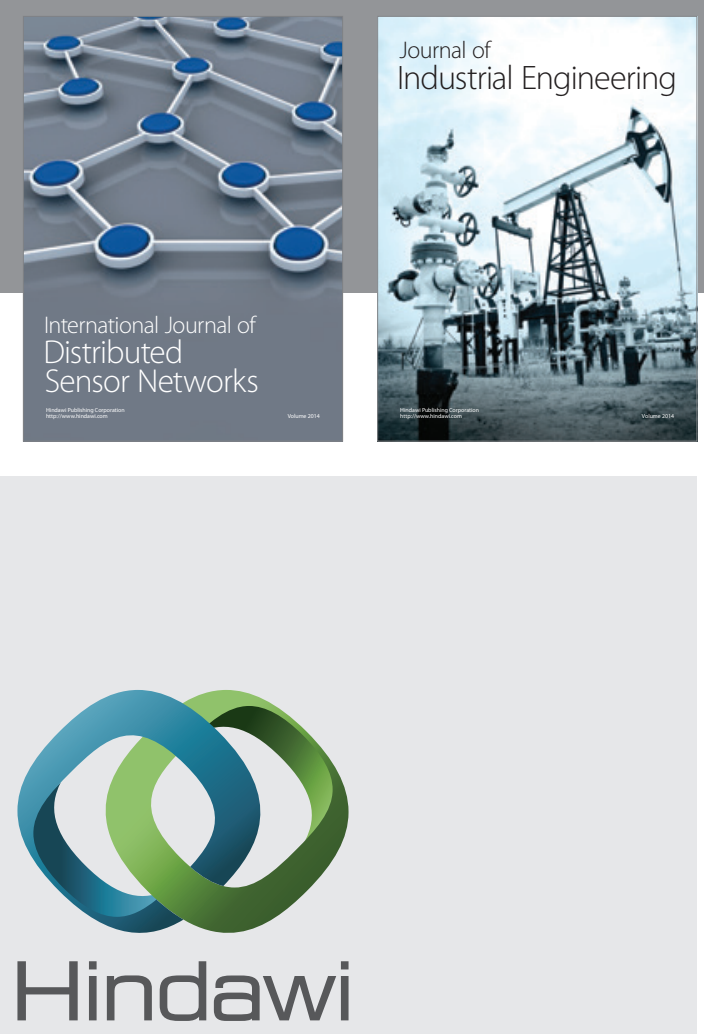

Submit your manuscripts at

http://www.hindawi.com

\section{Computer Networks} and Communications
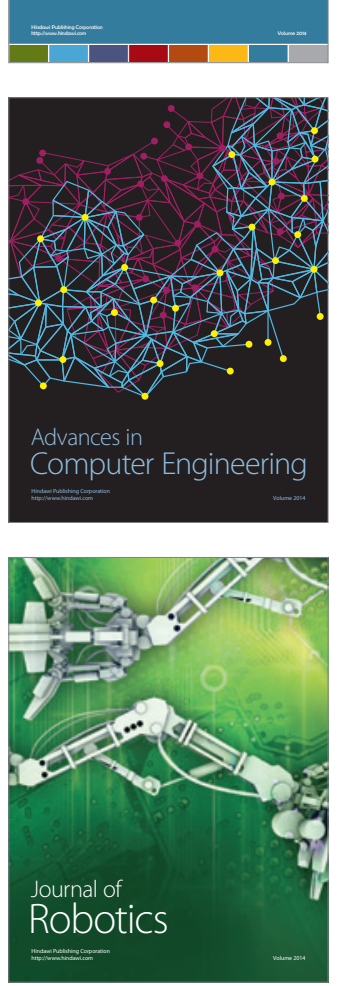
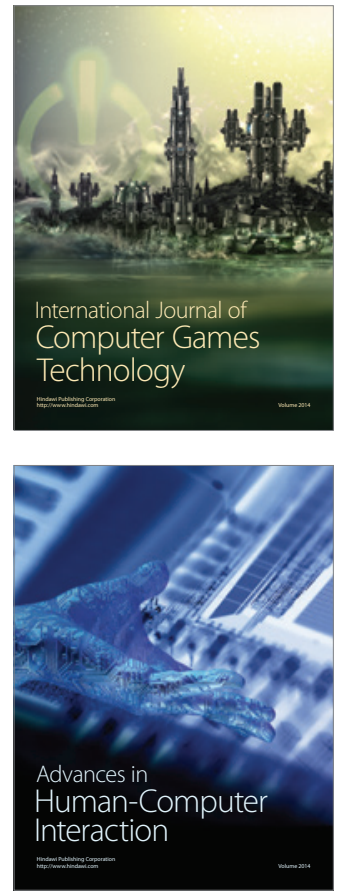
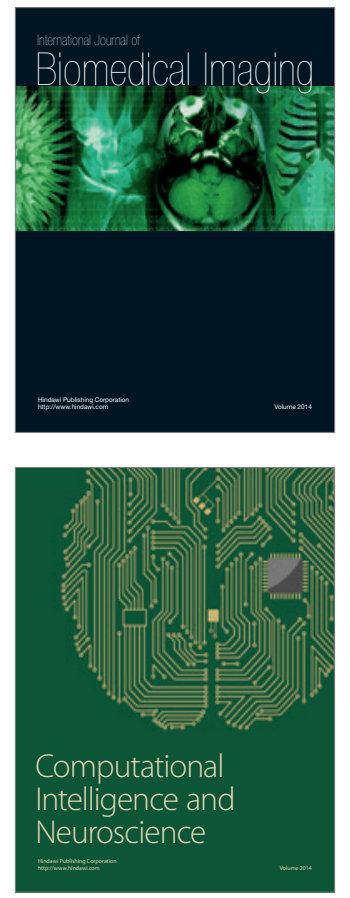
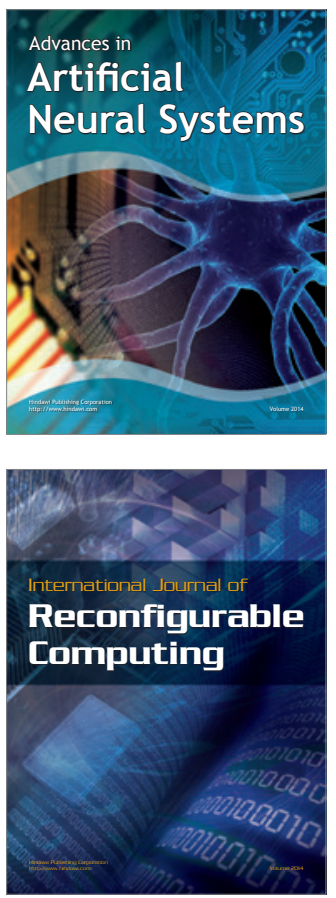
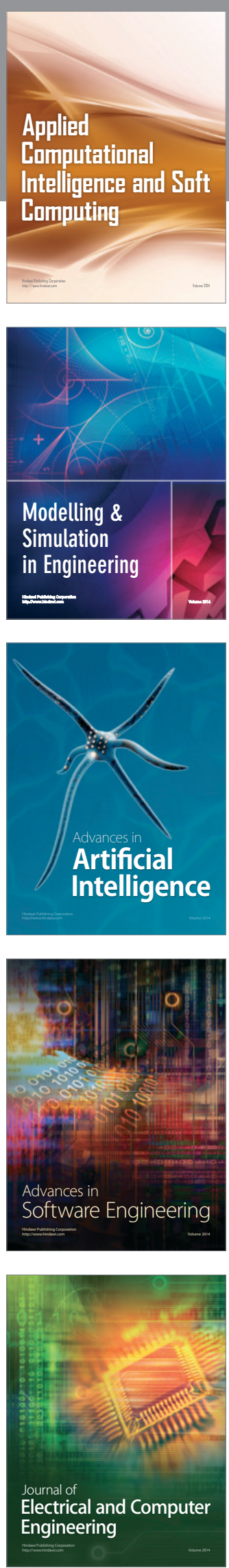